\title{
Patrick J. Bradley and Orlando Guntinas-Lichius: Salivary Gland Disorders and Diseases: Diagnosis and Management
}

\author{
Thieme Verlag, ISBN: 978-3-13-146491-0
}

\author{
Andreas Dietz
}

Received: 7 February 2012/Accepted: 12 February 2012/Published online: 30 September 2012

(C) Springer-Verlag 2012

Dear Ladies and Gentlemen,

It is my great honour to be asked to review the recently published book entitled: "Salivary Gland Disorders and Diseases: Diagnosis and Management" presented by the editors Patrick J. Bradley and Orlando Guntinas-Lichius.

Regarding the list of 52 contributors, the book is a very comprehensive work, counting all together 486 pages. The book is divided into six sections and due to this well organised in a perfect didactic line. The book covers all topics regarding salivary glands touching on physiology, diagnostics, pathology and current treatment of the diseases or disorders. Especially the section 2, paediatric disorders, is very interesting and shows a valuable collection of knowledge, which is in that way first time summarized in a very useful and informative way. Due to the fact that there are no big published series of, e.g., paediatric malignancies in salivary glands, the book describes all relevant bigger trials and retrospective case series and gives high-value practical recommendations for our daily work.

For me, it was really useful to see the differences between the adults and the children, even regarding operation procedures. Illustrations of operations are clear and many valuable tips of expired surgeons are given. All those statements focusing on neck dissection and adjuvant treatment are useful due to the fact that there are nearly no comparative controlled trials in the field. E.g., Peter Zbaeren and Kevin Harrington, both highly estimated specialists in the field, give their personal experience in differentiated handling of neck metastasis, chemotherapy and new therapeutics. The book ends with a short outlook from Vincent van der Poorten, who gives some ideas about new developments in molecular biology. After readings the book, especially this last chapter gives clear advices for future translational research and opens the gate for more interdisciplinary understanding of the multiple salivary gland disorders.

Inspired of the fact that the book is really comprehensive, the organisation is very nice, showing from outside colourbars which gives a lexicon like feature of the book. For me personally, reading the book was a great pleasure and I really learnt a lot. The book is mandatory for all doctors involved in the diagnosis and treatment of salivary gland disorders.

Congratulations to the editors for this really outstanding work.

A. Dietz is the Head of the Head and Neck Working group of the German ENT-Society

\footnotetext{
A. Dietz ( $\square)$

ENT-Clinic of University Hospital Leipzig, Leipzig, Germany

e-mail: hno@medizin.uni-leipzig.de

URL: http://hno.uniklinikum-leipzig.de
} 